\title{
Capacitive power transfer in biomedical implantable device: a review
}

\author{
Muhammad Zaki bin Mustapa, Shakir Saat, Yusmarnita Yusof, Muslimah Meor Shaari \\ Faculty of Electronic and Computer Engineering, Universiti Teknikal Malaysia, Malaysia \\ Centre for Telecommunication Research \& Innovation
}

\begin{tabular}{l}
\hline \hline Article Info \\
\hline Article history: \\
Received Sep 6, 2018 \\
Revised Nov 19,2018 \\
Accepted Feb 15, 2019 \\
\hline Keywords: \\
Biomedical implant devices, \\
Biosafety of wireless power \\
Transfer \\
Capacitive wireless power \\
Transfer \\
Class E inverter \\
\hline
\end{tabular}

\begin{abstract}
This paper presents the development of a new design method of capacitive power transfer (CPT) which is based on hybrid concept for Biomedical Implants. This method is able to improve various issues found in the widely used CPT system that is bipolar CPT method. Based on the ability of this purposed, the simulation of the CPT system has been designed to prove an amount of power transferred through a layer of tissue. The design used to validate the suggested model which to powering implanted device, and it was performed with $3 \mathrm{~cm}$ square plates, which have a layer of beef with the $5 \mathrm{~mm}$ thickness in between 2 coupling plate. Power signal was generated by Class E zero voltage switching. The Class E zero voltage switching has been designed to generating alternate current with the $1 \mathrm{MHz}$ frequency appropriate to the hybrid CPT system specification.
\end{abstract}

Copyright (c) 2019 Institute of Advanced Engineering and Science. All rights reserved.

Corresponding Author:

Muhammad Zaki bin Mustapa,

Faculty of Electronic and Computer Engineering,

Universiti Teknikal Malaysia, Melaka.

Email:m.zaki5377@gmail.com

\section{INTRODUCTION}

Wireless power transfers have been broadly explored for various applications such as electric car charging, smartphone charger, RFID devices, powering implantable devices and much more [1]. Wireless power transfers approach generally can be classified into near field and far field power transfer applications. The near-field approach can be divided into several types which are an inductive resonance frequency coupling (IRFC), ultrasonic energy transfer, and near-field capacitive coupling (NCC). Previously, the comparison of characteristics each type of wireless power transfers have been made by other researchers. Based on comparison made, every type of wireless power transfers has the advantage over other types [2]. This reason reinforces that each type of WPT can be applied according to suitability and requirement of the biomedical implantable device.

Advancement of biomedical implants has been successful in the creation of several types of implantable devices such as cardiovascular implantable devices, Cochlear, neural, retinal and more others to meet medical needs. Each type of implantable device has its own specifications. Some implantable device types require a biosafety source of electrical power to operate properly. The sealed battery as the power source of implantable devices can only supply the energy for a limited time. The patient will experience trauma with repeated surgery to replacing the battery. Because of constant dependence on electrical power sources, a wireless power transfer method is required to overcome the limited lack of battery power source. Nowadays, biomedical technology already established with both type wireless power transfer, which is inductive and ultrasound power transfer, and already used for wireless power delivery for several implantable devices such as neural implants. However, both methods have weaknesses in terms of signal interference [3].

Since the inductive power transfer propagating power by inducing electromagnetic field, it is risky to interfere with other surrounding devices that sensitive to the electromagnetic field. Thus, reducing this 
kind of interference is one of the main challenges to designing inductive power transfers [4]. The capacitive wireless power transfer has been used in several applications and already proposed for the biomedical implantable device since 2009 by Amir M. Sodagar et. al [5]. This approach was also recently used for sensing biomedical signal in [6]. The achievement in capacitive linkage studies is not as successful as the inductive link. This is because of the limited range factor of the capacitive link. NCC approaches different to the inductive power transfer, in which the electromagnetic as a mechanism for transferring energy. But In CPT, the electric field between both parallel transmitters and receiver plates is used as the mechanism to transferring energy in term of power and data. It was the solution to the IPT problem which the CPT approaches eliminated the relatively large electromagnetic interference from the sensitive electronic implantable device [7].

Thus, the main objective of this paper is to prepare a comprehensive review of CPT based on biomedical implantable device application. The review of capacitive coupling power transfer of biomedical device will assist to understand the principle, requirement, and limitation of the design. The parameter of capacitive coupling power transfers should be designed following the requirement of devices and the biosafety specification standard. To build the Capacitive Power Transfer in Biomedical Implantable Device, the Power Management of capacitive coupling system must be known perfectly [8].

Contents of this paper are as follows: In section 2, the basic theory of the capacitive coupling power transfer in biomedical device is explained, and it continues to Biosafety CPT mechanism in subsection A. The Power management is explained in section 3 and it continues to power transfers efficiency of class $\mathrm{E}$ and medium of data telemetry in subsection A and B respectively. The drawbacks and solution of the system are discussed in section 4. The paper summarizes the research needs of capacitive wireless power transfer in biomedical implantable device's technology. In order to improve the system, the study remains open to discover the best solution. Thus, the author prepares several planning for future work for these research and development of CPT in biomedical implantable device.

\section{CAPACITIVE COUPLING POWER TRANSFER IN BIOMEDICAL DEVICE}

CPT approaches is a wireless power transfer scheme using pair of coupling plate to transfer electrical field through a dielectric barrier. This scheme is performed by the displacement of current without a conductor as a medium for the transfer of charges. Refer to Figure 1 for the CPT schematic as an illustration application of biomedical implantable devices. The powering system consists pairs of conductor plate located on either side of the skin of certain tissue thickness, and its form a capacitive coupling which to enable the wireless power transfer [9].

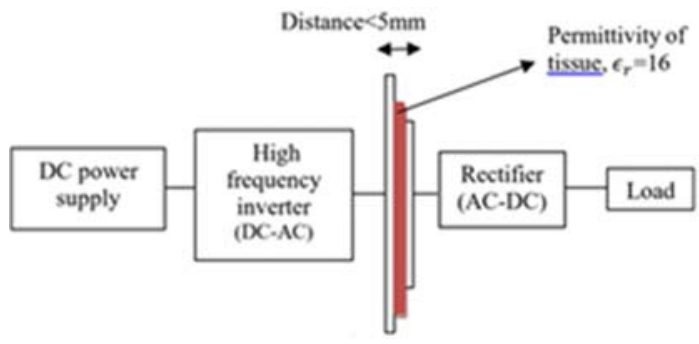

Figure 1. Pair coupling plate for implantable devices

The principle of the CPT wireless power transfer is about the displacement current to transfer the power between two contactless conductor plates. Together with that, conduction current also induced between both plates. Both displacement and conduction equation is given in (1,) and (2), respectively. [10].

$$
\begin{aligned}
& I_{\text {disp }}=\epsilon_{0} \epsilon_{r}(\omega) A \frac{\partial \vec{E}}{\partial t} \\
& I_{\text {cond }}=\frac{V(t) \sigma(\omega) A}{D}
\end{aligned}
$$

For a complete circuit of power delivery to load, two such capacitances are required. In order to improve the effectiveness power delivery, displacement current should be increased while the conduction 
current needs to minimize as low as possible to reduce the power loss with considering the parameter of A, E, $\mathrm{D}$ and $\epsilon_{-} \mathrm{r}$ in optimum values [11]. From the displacement current in (1), there are several parameters can increase the displacement current and at the same time to improve the power transfer efficiency. The parameter is electric field strength, $\mathrm{E}$ is most affecting the displacement current value. These parameters can improve the efficiency by increasing the transmitter excitation voltage and reducing the separation distance between the coupling plates. The displacement current value is also affected by the rate of change of electric field. Thus the efficiency can be improved by increasing the operating frequency. Besides that, increasing the magnitude of electric field also can improve the transferring power efficiency, whereby it can be done by expanding the conductor of coupling plate area [12]. However, all the parameters were limited by the biomedical implantable device specification.

Since the pairs of conductor plate (limited Biohazard dimension) separated D by a few millimeter tissues $(<5 \mathrm{~mm})$ as the dielectric medium $\epsilon_{-} r, \sigma$, the power transfer strength is limited due to the weak capacitance values [13]. Thus, few millimeters deep of tissues which possible to attach the receiver plate is under the layer of skin as illustrate in Figure 2 [1].

Besides that, the surface area of the coupling plate that needs to be minimized will be a challenge to obtain high power transfer values. Thus, the specific on designing capacitive wireless power transfer should be proposed to overcome the constraints and to fulfill the biomedical implantable device requirement.

In the designing capacitive wireless power transfer for biomedical purpose, there are several particular aspect needs to be concerned, such as the element of Biosafety, the performance of the power delivery efficiency, range for misalign, effective range of system and others [14].

\section{RESULTS AND ANALYSIS}

In this section, it is explained the results of research and at the same time is given the comprehensive discussion. Results can be presented in figures, graphs, tables and others that make the reader understand easily $[2,5]$. The discussion can be made in several sub-chapters.

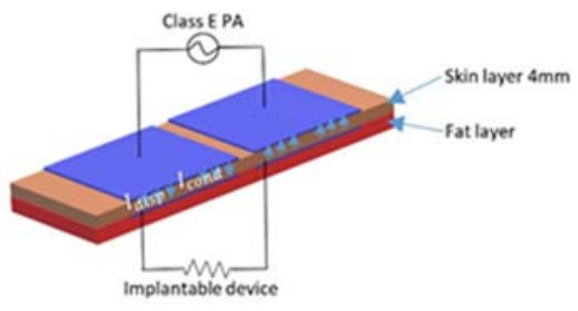

Figure 2. Pair coupling plate for implantable devices

\subsection{Biosafety CPT mechanism}

The international commission on non-ionizing radiation protection (ICNIRP) identify clearly maximum limits of measurement specific absorption rate (SAR) ordinary over $10 \mathrm{~g}$ of tissue limited to 2 $\mathrm{W} / \mathrm{kg}$ for exposure $[15,16]$.

$$
S A R=\frac{\sigma}{2 \rho}|E|^{2}
$$

Where,

$\sigma=$ Conductivity

$\rho=$ Density of the tissue

$\mathrm{E}=$ Electric field strength

The Biosafety comparative wireless power transfer has been proposed in deep by Ahmed I. AlKalbani et.al [4].The result is summarized in Table 1. Table 1 shows an experiment of maximum 10-g averaged SAR as a function of lateral distance in $\mathrm{mm}$ and the transmitted power is $1 \mathrm{~W}$ at $5 \mathrm{MHz}$ for both capacitive and inductive power transfer [17]. The investigation proved that the electromagnetic simulations illustrated that a powering scheme utilizing capacitive coupling yields smaller 10-g SAR values compared to inductive coupling. This is because, in case of capacitive coupling, the transmitted RF energy remains evenly confined in the field between the capacitor plates, while the inductive power transmission, the RF spreads out including focused points where the E-Field is at its highest [12]. However, the efficiency of capacitive

Capacitive power transfer in biomedical implantable device: A review (Muhammad Zaki bin Mustapa) 
coupling link performance was not favorable as a function of the gap between plates compared to inductive coupling. Therefore, various studies have been conducted to improve power transmission efficiency $[18,19]$.

Table 1. Result of SAR due to distance of capacitive and inductive analysis [4]

\begin{tabular}{ccc}
\hline Distance $(\mathrm{mm})$ & $\begin{array}{c}\text { Rate of absorption (peak 10g } \\
\text { SAR) Capacitive plate, }\end{array}$ & $\begin{array}{c}\text { Rate of absorption (peak 10g } \\
\text { SAR) Inductive coil, }\end{array}$ \\
\hline 1 & 1.52 & 2.44 \\
3 & 1.50 & 2.42 \\
5 & 1.65 & 2.40 \\
7 & 1.57 & 2.38 \\
9 & 1.44 & 2.34 \\
11 & 1.26 & 2.29 \\
13 & 1.10 & 2.24 \\
\hline
\end{tabular}

The result of SAR analysis will be different if the parameter of internal capacitance of the coupling plate was changing and it resulted in different value of electric field strength. Analysis to determine relationship size of the capacitor plate with SAR value has been described by Rangarajan Jegadeesan et, al [20]. A simulation using CST software has been done to investigate in ICNIRP requirement for three different sizes of capacitor coupling. The articles summarize larger size plate with $250 \mathrm{~mW}$ power transmitter is safe regarding to an international standard compared to smaller plate size. In this article they run a simulation of $20 \mathrm{mmX} 20 \mathrm{~mm}, 15 \mathrm{mmX} 20 \mathrm{~mm}$, and $10 \mathrm{mmX} 20 \mathrm{~mm}$ sized of plates. Thus, it found that the $20 \mathrm{~mm}$ size plate with $250 \mathrm{~mW}$ transmission power was safe according to international standards. Furthermore, the large size of the plate will be able to obtain better transmission power efficiency rates[21, 22].

\subsection{Power management}

The previous section, the limitation on the CPT design based on the Biosafety constraint has been discussed. The limitation will be a part of modeling and experimental validation CPT as a developing wireless power transfer to powering biomedical implantable devices. Determining the size of the capacitance plate at receiver part is very important before the power management design is proposed. Since the receiving plate will be placed inside after the skin layer, the size of the plate should minimize as small as possible while small capacitance plate will cause the reduction of the power transmission efficiency [23]. However, it can be solved by choosing the optimum operating frequency and apply the appropriate impedance matching network of the power amplifier system will be increasing the power transmission efficiency while ensuring high frequency generated in perfect zero voltage switching. [24].

The study of optimization for capacitive wireless power transfers system has been covered by many researchers [25]. The optimization power transfers study focuses on increasing the efficiency of power transfers and to manage system due to misalignment of coupling plate. The power transmission efficiency rate for CPT depends on power signal frequency generated by the inverter. An Inverter or known as Power amplifier topologies has been classified by its design named $\mathrm{A}, \mathrm{B}, \mathrm{AB}$ and $\mathrm{C}$ for analog designs and class $\mathrm{D}$ and $\mathrm{E}$ for switching design. In [26] has recommended class $\mathrm{E}$ amplifier as its proof ability to give the highest efficiency with simple switching circuit required. The class E power amplifier has two different types which are a Class E zero current switching and Class E zero voltage switching where both belong to the family of soft-switching inverters. Class E ZVS inverters known as the most efficient inverter which the switch closed at zero voltage conditions occurred if all components values of properly designed. General Class E zero voltage switching an amplifier with CPT links schematic diagram as shown in Figure 3 [27].

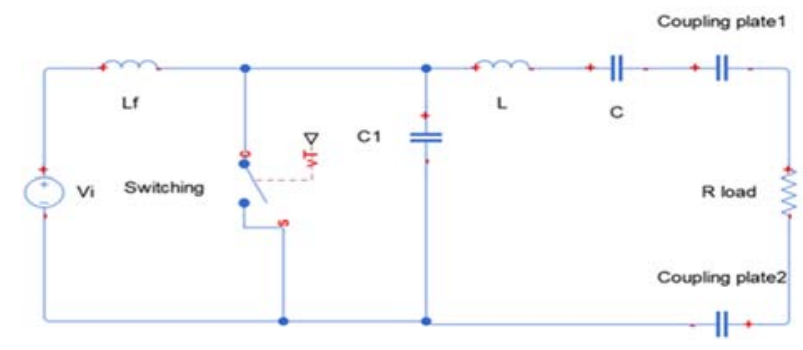

Figure 3. Environments condition for traffic monitoring

Int J Pow Elec \& Dri Syst, Vol. 10, No. 2, June 2019 : 935 - 942 
The Class E zero voltage switching circuit basically consists of an MOSFET as a switching component, a L-C-R series resonant circuit, a shunt capacitor, and a choke inductor. The switching and also determined as the driver which about turning on and off at the operating frequency $f=\omega /(2 \pi)$. The choke inductor is used to terminate the AC ripple on the DC supply current, thus the value of choke inductor required to be high enough for that. The $\mathrm{C} 1$ will be in a short circuit condition and the state cause the resonant circuit consist of $\mathrm{L}, \mathrm{C}$, and RI when the switch in ON state. While the switch in OFF state, the resonant circuit will consists of $\mathrm{C} 1, \mathrm{~L}, \mathrm{C}$ and $\mathrm{Ri}$ in series connection. Since $\mathrm{C} 1$ and $\mathrm{C}$ are in series, the equivalent capacitance will be lower than both value $C 1$ and $C, C e q=C C 1 /(C+C 1)[19]$. The output will have traits with two difference resonant frequencies and two differences loaded quality factors. The equation is as follows[28,29]. When the switch is ON:

$$
f_{o 1}=1 /(2 \pi \sqrt{L C}) \text { and } Q_{L 1}=\omega_{o 1} L / R_{i}=1 /\left(\omega_{o 1} C R_{i}\right)
$$

When the switch is OFF:

$$
f_{o 2}=1 /\left(2 \pi \sqrt{L C} C_{1} /(C+)\right)
$$

The L-C-Ri series resonant circuit will be representing an inductive load, if the frequency $\mathrm{f}$ is greater than the resonant frequency f01. Thus inductance $\mathrm{L}$ can be divided into two La and Lb, as shown in Figure 4, La resonates with $\mathrm{C}$ at the operating frequency and expressed by

$$
\omega=\frac{1}{\sqrt{L_{a} C}}
$$

Then the quality factor loaded can be expressed as

$$
Q_{L}=\frac{\omega L}{R_{i}}=\frac{\omega\left(L_{a}+L_{b}\right)}{R_{i}}=\frac{1}{\omega C R_{i}}+\frac{\omega L_{b}}{R_{i}}
$$

There are several specifications on the class e amplifier design in order to achieve optimal values of high frequency power supplies. Such specification is as follows [30].

a. The value of an inductor after DC power supply should be high to avoid the alternating current turn back to the DC power supply.

b. The quality factor of the load is in the highest to make sure the load current as high frequency sinusoidal in consistent condition.

c. The switching operation generates zero ON and OFF state laws. Every parameter of parasitic resistance values is neglected.

\subsection{Power transfers efficiency of class $E$}

The power efficiency of class e estimation can be determined from the value of total power loss (P_LS) in each circuit component. Hence, power loss considered to consist of

a. Power loss of choke inductor (Lf)

b. Power loss of switch conduction loss (Ds)

c. Power loss of shunting capacitor (C1)

d. Power loss of resonant inductor (L)

e. Power loss of resonant capacitor (C)

f. Power loss at the fall time (tf)

Thus, the efficiency of the class E power amplifier can be written as

$$
\eta_{I}=\frac{P_{R i}}{P_{R i}+P_{L S}}
$$

However, there is also an element of input Zin and load ZL impedance equilibrium that needs to be ascertained to produce the maximum power at the output of the class e power amplifier. Figure (4) illustrates the impedance matching [25]. 


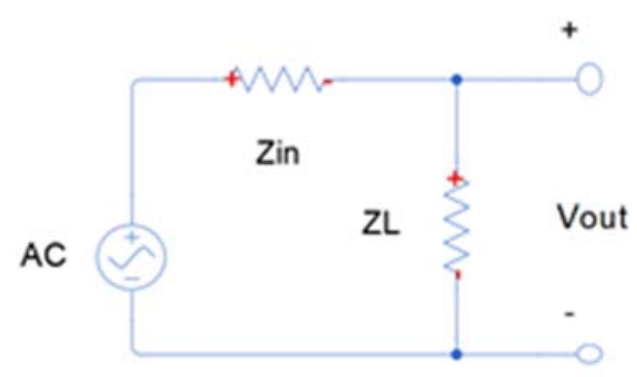

Figure 4. Impedance matching $(\mathrm{Zin}=\mathrm{ZL})$

The application impedance matching on ZVS Class E power amplifier has been discussed by Yusmarnita Yusop et.al (2016) [30]. The author describes the way to design the Class-E amplifier with an impedance matching circuit $\pi \mathrm{lb}$. Compensation topologies of high-power wireless power transfer systems has been proposed by Wei Zhang (2016) [31]. The author reviewed several types of impedance matching topologies and specialized function. However, both coupling plate impedance should be involved in designing the impedance matching circuit. Thus, the coupling plate which applied need to express into a modeling and considered to get the optimum output power in the delivery. The coupling plate modelling has been done [32].

Besides that class e power amplifier also have the limitation on the operating frequency. It is because, when the power amplifier designed for high operating frequency, it will increase the capacitance of mosfet output and become higher than the required to operate zero voltage switching. Thus, the maximum operating frequency value for $50 \%$ duty cycle is given by [32]

$$
f_{\text {max }}=\frac{0.1971}{2 \pi R_{i} C_{\text {out }}}
$$

Therefore the choice of the MOSFET component must take into account the value of the output capacitance, in which the low output capacitance is more capable of operating at higher frequencies switching operation. But it still restricts operating frequencies, except when making modifications to switching class E amplifier circuits.

\subsection{Medium of data telemetry}

Data transfer is one of the main parts of the implantable device, in particular the type of monitoring and controlling devices. The main thing emphasized in telemetry data is the ability of data to be transmitted without interruption and the accuracy of the data received to the receiver circuit. Thus, the study of data transfer application of capacitive wireless transfer also should be covered. Amir M. Sodagar and Parviz Amiri [2] has been proposed a method for power and data wireless transfer using capacitive plate. The authors proposed two different sets of coupling capacitor plate which to transmit power and data respectively, while this approach will lead to more tissue losses.

\subsection{Drawbacks and solution medium}

Power transfer efficiency PTE is an important value which to ensuring the final load receive enough power for their circuit operation. Since the CPT efficiency very sensitive to changes in the alignment of coupling capacitive plates, the power delivery on the receiver will be inconsistence value. This is a challenge for researchers to find solutions to the inconsistent problems of power output.

The auto-tuning system has been proposed for the capacitive power transfer by Kai Lu and Sing Kiong Nguang [33] to solve an inconsistent output power. The author stated that the changes in the alignment of coupling capacitive plate which replace the load of class e power amplifier will cause the increment the switching loss of that system, and the efficiency of the system will drop significantly. Thus, a linear quadratic Gaussian (LQR) controller has been proposed to adjust the operating frequency to maintaining the receiver output voltage. However, the system approached require the output voltage value at receiver part as the controller respond while most auto tuning controller unit placed on transmitter part. Thus, this system demands another wireless communication to operate the controller.

Reza Erfani et.al (2017) [34] has been using small magnets to cater the misalignment and also propose the larger transmitter plate to cover losses due the misalignment with small receiver plate. In order to improve the efficiency of changing power transferred, the study remains open to discover the best solution.

Int J Pow Elec \& Dri Syst, Vol. 10, No. 2, June 2019 : 935 - 942 
The improvement can be studied through several parts of the system, such as the feedback and regulating the operating frequency, design of impedance matching, and design of coupling capacitive plate [35]. Thus, an efficient and reliable way of capacitive wireless power transfer for implantable biomedical devices is expected as a successful outcome.

\section{CONCLUSION}

This paper is to prepare a comprehensive review about CPT based on biomedical implantable device application based on the previous researchers report. Based on this study, capacitive power transfer systems are a new method that has potential to apply to biomedical implantable devices. It was the solution to the IPT problem which the CPT approaches eliminated the relatively large electromagnetic interference from the sensitive electronic implantable device. The principle of the CPT wireless power transfer is about the displacement current to transfer the power between two contactless conductor plates. The parameter is electric field strength, $\mathrm{E}$ is most affecting the displacement current value. However, all the parameters were limited by the biomedical implantable device specification. The Biosafety comparative wireless power transfer has been proposed in deep by Ahmed I. Al-Kalbani et.al [4]. The investigation proved that the electromagnetic simulations illustrated that a powering scheme utilizing capacitive coupling yields smaller 10 -g SAR values compared to inductive coupling. Class E ZVS inverters known as the most efficient inverter which the switch closed at zero voltage conditions occurred if all components values of properly designed. However, there is also an element of input Zin and load ZL impedance equilibrium that needs to be ascertained to produce the maximum power at the output of the class e power amplifier. Since the CPT efficiency very sensitive to changes in the alignment of coupling capacitive plates, the power delivery on the receiver will be inconsistence value. In order to improve the efficiency of changing power transferred, the study remains open to discover the best solution. The improvement can be studied through several parts of the system, such as the feedback and regulating the operating frequency, design of impedance matching, and design of coupling capacitive plate. However, there are so many things has to master in, especially on the standard of procedure of the designing capacitive wireless power transfer for biomedical practice. Thus, the author prepares several planning for future work for these research and development.

The development of capacitive power transfer in biomedical implantable device should have their specific modelling which is to considering every parameter which effecting the whole operating system. The modelling was include the power management circuit, coupling capacitor with tissue, and the receiver part with implant device as their load. To design the system, all the limitation in term of biosafety and the device requirement should be followed. To ensure the vision of bio electronic medicine technology yet to come, the path for the inventor advancements in capacitive wireless power transfer is becoming clearer.

\section{ACKNOWLEDGEMENTS}

The authors would like to thank Universiti Teknikal Malaysia Melaka (UTeM) and Ministry of Science, Technology and Innovation for sponsoring this work under the Science fund grant (06-01-14-SF0138L00030).

\section{REFERENCES}

[1] K. Agarwal, R. Jegadeesan, Y. X. Guo, and N. V. Thakor, "Wireless Power Transfer Strategies for Implantable Bioelectronics," IEEE Reviews in Biomedical Engineering, vol. 10. pp. 136-161, 2017.

[2] A. M. Sodagar and P. Amiri, "Capacitive coupling for power and data telemetry to implantable biomedical microsystems," in 2009 4th International IEEE/EMBS Conference on Neural Engineering, NER '09, pp. 411-414, 2009.

[3] D. Rozario, N. A. Azeez, and S. S. Williamson, "Analysis and design of coupling capacitors for contactless capacitive power transfer systems," in 2016 IEEE Transportation Electrification Conference and Expo, ITEC 2016, 2016.

[4] A. I. Al-Kalbani, M. R. Yuce, and J. M. Redoute, "A biosafety comparison between capacitive and inductive coupling in biomedical implants," IEEE Antennas Wirel. Propag. Lett. vol. 13, pp. 1168-1171, 2014.

[5] A. M. Sodagar and K. Najafi, "Wireless interfaces for implantable biomedical microsystems," in Midwest Symposium on Circuits and Systems, vol. 2, pp. 265-269, 2006.

[6] M. Takhti, F. Asgarian, and A. M. Sodagar, "Modeling of a capacitive link for data telemetry to biomedical implants," in 2011 IEEE Biomedical Circuits and Systems Conference, BioCAS 2011, pp. 181-184, 2011.

[7] Fariborz Musavi; Murray Edington; Wilson Eberle, "Wireless power transfer: A survey of EV battery charging technologies", in IEEE Energy Conversion Congress and Exposition (ECCE), 2012, pp. 1804-1810.

[8] R. Jegadeesan, K. Agarwal, Y.-X. Guo, S.-C. Yen, and N. V. Thakor, "Wireless Power Delivery to Flexible Subcutaneous Implants Using Capacitive Coupling," IEEE Trans. Microw. Theory Tech., vol. 65, no. 1, 2017. 
[9] Quan Xiong1, "Wireless Charging Device for Artificial Cardiac Pacemaker", International Conference on Information Technology and Management Innovation (ICITMI), Atlantis Press, pp. 765-768, 2015.

[10] Chao Liu; Aiguo Patrick Hu; Nirmal-Kumar C. Nair, "Coupling study of a rotary Capacitive Power Transfer system", in IEEE International Conference on Industrial Technology, 2009, pp. 1-6.

[11] Hua Zhang; Fei Lu; Heath Hofmann; Weiguo Liu; Chris Mi, "A Large Air-Gap Capacitive Power Transfer System with a 4-Plate Capacitive Coupler Structure for Electric Vehicle Charging Applications" in IEEE Applied Power Electronics Conference and Exposition (APEC), pp. 1726 - 1730, 2016.

[12] M. Kline, "Capacitive Power Transfer," Report, pp. 1-35, 2010.

[13] R. Jegadeesan, K. Agarwal, Y.-X. Guo, S.-C. Yen, and N. V. Thakor, "Wireless Power Delivery to Flexible Subcutaneous Implants Using Capacitive Coupling," IEEE Trans. Microw. Theory Tech., pp. 1-13, 2016.

[14] L. Huang; A. P. Hu, "Defining the mutual coupling of capacitive power transfer for wireless power transfer", in IET Journals \& Magazines, Electronics Letters, 22nd October, Vol. 51 No. 22 pp. 1806-1807, 2015.

[15] K. Ramrakhyani, S. Mirabbasi, Mu Chiao, and C. M, "Design and optimization of resonance-based efficient wireless power delivery systems for biomedical implants," IEEE Trans. Biomed. Circuits Syst., vol. 5, no. 1, pp. 48-63, 2011.

[16] "Summary of blood brain barrier (BBB) permeability", in IEEE Standard for Safety Levels with Respect to Human Exposure to Radio Frequency Electromagnetic Fields, 3 kHz to $300 \mathrm{GHz}$, IEEE Std C95.1-2005.pp. 57, 2005.

[17] J. Dai and D. C. Ludois, "A Survey of Wireless Power Transfer and a Critical Comparison of Inductive and Capacitive Coupling for Small Gap Applications," IEEE Trans. Power Electron., vol. 30, no. 11, pp. 6017-6029, 2015.

[18] Vladimir Vulfin, Shai Sayfan-Altman \& Reuven Ianconescu, "Wireless power transfer for a pacemaker application". Journal of Medical Engineering \& Technology, 2017.

[19] M. Kline, I. Izyumin, B. Boser, and S. Sanders, "Capacitive power transfer for contactless charging," in Conference Proceedings - IEEE Applied Power Electronics Conference and Exposition - APEC, 2011, pp. 1398-1404.

[20] R. Jegadeesan, Y. X. Guo, and M. Je, "Electric near-field coupling for wireless power transfer in biomedical applications," in 2013 IEEE MTT-S International Microwave Workshop Series on RF and Wireless Technologies for Biomedical and Healthcare Applications, IMWS-BIO 2013 - Proceedings, 2013.

[21] R. Erfani, F. Marefat, A. M. Sodagar, P. Mohseni, "Modeling and experimental validation of a capacitive link for wireless power transfer to biomedical implants", IEEE Transactions on Circuits and Systems II: Express Briefs, 2017.

[22] R. Jegadeesan, K. Agarwal, Y.-X. Guo, S.-C. Yen, and N. V. Thakor, "Wireless Power Delivery to Flexible Subcutaneous Implants Using Capacitive Coupling," IEEE Trans. Microw. Theory Tech., vol. 65, no. 1, pp. 280 292, 2017.

[23] M. P. Theodoridis, "Effective capacitive power transfer," IEEE Trans. Power Electron., vol. 27, no. 12, pp. 49064913, 2012.

[24] T. M. Mostafa, A. Muharam, and R. Hattori, "Wireless battery charging system for drones via capacitive power transfer," in 2017 IEEE PELS Workshop on Emerging Technologies: Wireless Power Transfer, WoW 2017, 2017.

[25] J. Kim, D. H. Kim, and Y. J. Park, "Analysis of Capacitive Impedance Matching Networks for Simultaneous Wireless Power Transfer to Multiple Devices," IEEE Trans. Ind. Electron., vol. 62, no. 5, pp. 2807-2813, 2015.

[26] J. J. Casanova, Z. N. Low, and J. Lin, "Design and optimization of a class-E amplifier for a loosely coupled planar wireless power system,” IEEE Trans. Circuits Syst. II Express Briefs, vol. 56, no. 11, pp. 830-834, 2009.

[27] H. Zhang, F. Lu, H. Hofmann, W. Liu, and C. C. Mi, "A Four-Plate Compact Capacitive Coupler Design and LCLCompensated Topology for Capacitive Power Transfer in Electric Vehicle Charging Application," IEEE Trans. Power Electron., vol. 31, no. 12, pp. 8541-8551, 2016.

[28] Vrushali B. Gore, Dhanashri H. Gawali, "Wireless Power Transfer Technology for Medical Applications". In IEEE Conference on Advances in Signal Processing (CASP), 2016, pp. 455-460.

[29] D. C. Ludois, J. K. Reed, and K. Hanson, "Capacitive power transfer for rotor field current in synchronous machines," IEEE Trans. Power Electron., vol. 27, no. 11, pp. 4638-4645, 2012.

[30] Y. Yusop, S. Saat, Z. Ghani, H. Husin, and S. K. Nguang, "Capacitive power transfer with impedance matching network," in 2016 IEEE 12th International Colloquium on Signal Processing \& Its Applications (CSPA), 2016, pp. $124-129$.

[31] Y. Yusop, S. Saat, S. K. Nguang, H. Husin, and Z. Ghani, "Design of Capacitive Power Transfer Using a Class-E Resonant Inverter," J. Power Electron., vol. 16, no. 5, pp. 1678-1688, 2016.

[32] W. Zhang and C. C. Mi, "Compensation topologies of high-power wireless power transfer systems," IEEE Trans. Veh. Technol., vol. 65, no. 6, pp. 4768-4778, 2016.

[33] K. Lu and S. K. Nguang, "LQG control of capacitive power transfer system," in 2017 IEEE PELS Workshop on Emerging Technologies: Wireless Power Transfer, WoW 2017, 2017, pp. 128-132.

[34] R. Erfani, F. Marefat, A. M. Sodagar, P. Mohseni, "Modeling and experimental validation of a capacitive link for wireless power transfer to biomedical implants", IEEE Transactions on Circuits and Systems II: Express Briefs, 2017

[35] R. Erfani, F. Marefat, A. M. Sodagar, and P. Mohseni, "Transcutaneous capacitive wireless power transfer (CWPT) for biomedical implants," in Proceedings - IEEE International Symposium on Circuits and Systems, 2017.

Int J Pow Elec \& Dri Syst, Vol. 10, No. 2, June 2019 : 935 - 942 